\title{
AN IDENTITY FOR A CLASS OF ARITHMETICAL FUNCTIONS OF TWO VARIABLES
}

\section{J. CHIDAMBARASWAMY and P.V. KRISHNAIAH}

\author{
Department of Mathematics \\ The University of Toledo \\ Toledo, Ohio 43606
}

(Received November 17, 1986)

ABSTRACT. For a positive integer $r$, let $r_{*}$ denote the quotient of $r$ by its largest squarefree divisor $\left(1_{\star}=1\right)$. Recently, K. R. Johnson proved that

$$
\begin{aligned}
& \text { (*) } \quad \sum|\mathrm{C}(\mathrm{d}, \mathrm{r})|=\mathrm{r}_{*} \pi(\mathrm{a}+1) \pi \quad(\mathrm{a}(\mathrm{p}-1)+1) \text { or } 0 \\
& \mathrm{~d}\left|\mathrm{n} \quad \mathrm{p}^{\mathrm{a}}\right|\left|\frac{\mathrm{n}}{\mathrm{r} .} \quad \mathrm{p}^{\mathrm{a}}\right| \mid \frac{\mathrm{n}}{\mathrm{r}_{*}} \\
& p+r \quad p \mid r
\end{aligned}
$$

according as $r^{*} \mid n$ or not where $c(n, r)$ is the well known Ramanujan's sum. In this paper, using a different method, we generalize $(*)$ to a wide class of arithmetical functions of 2 variables and deduce as special cases (*) and similar formulae for several generalizations of Ramanujan's sum.

KEY WORDS AND PHRASES. Arithmetical functions of two variables and multiplicativity in both variables. Ramanujan's sum and its generalizations.

1980 AMS SUBJECT CLASSIFICATION CODE. 10A20.

1. INTRODUCTION .

For a positive integer $r$, let $r_{*}$ denote the quotient of $r$ when divided by its largest square free divisor $\left(1_{*}=1\right)$. Recently $K$. R. Johnson [1] proved that

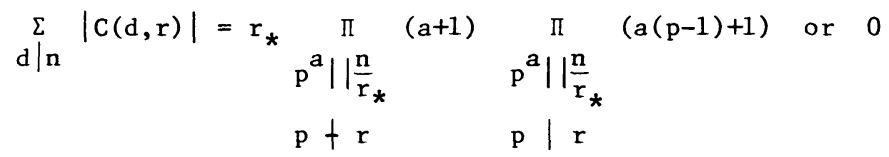

according as $r_{*} \mid n$ or not, where $C(n, r)$ is the well known Ramanujan's sum and $p^{a}|| n$ means that $\mathrm{p}^{\mathrm{a}} \mid \mathrm{n}$ and $\mathrm{p}^{\mathrm{a}+1}+\mathrm{n}$. In his proof, since $\mathrm{c}(\mathrm{n}, \mathrm{r})$ is not multiplicative in $n$, he used [1] two identities concerning $C(n, r)$ that were proved by him in an earlier paper [2]. As a matter of fact (1.1) can be obtained directly from the well known property of the Ramanujan's sum, namely its multiplicativity in both variables $n$ and $r$. In section 2, using this method, we generalize (1.1) (see theorem) to a class of arithmetical functions of two variables and in section 3, specializing our theorem, we deduce as corollaries, formulae analoguus to (1.1) for several generalizations of the Ramanujan's sum and (1.1) also.

We recall that an arithmetical function $f(n, r)$ is said to be multiplicative in both variables $n$ and $r$ if

$$
\begin{aligned}
\left(n_{1}, n_{2}\right)=\left(r_{1}, r_{2}\right)=\left(n_{1},\right. & \left.r_{2}\right)=\left(n_{2}, r_{1}\right)=1 \text { implies } \\
& f\left(n_{1} n_{2}, r_{1} r_{2}\right)=f\left(n_{1}, r_{1}\right) f\left(n_{2}, r_{2}\right)
\end{aligned}
$$


and that such a function is completely determined by its values $f\left(p^{a}, p^{b}\right)$ for primes $p$ and non negative integers $a$ and $b$.

2. MAIN THEOREM.

For given arithmetical functions $g(n)$ and $h(n)$ and for a given positive integer $k$, let

$$
\mathrm{S}^{(\mathrm{k})}(\mathrm{n}, \mathrm{r})=\mathrm{S}_{\mathrm{g}, \mathrm{h}}^{(\mathrm{k})}(\mathrm{n}, \mathrm{r})=\sum_{\mathrm{d}^{\mathrm{k}}} \sum_{\left(\mathrm{n}, \mathrm{r}^{\mathrm{k}}\right)_{\mathrm{k}}} \mathrm{g}(\mathrm{d}) \mu\left(\frac{\mathrm{r}}{\mathrm{d}}\right) \mathrm{h}\left(\frac{\mathrm{r}}{\mathrm{d}}\right)
$$

where $\mu(n)$ is the well known Möbius function and $(x, y)_{k}$ stands for the greatest common $k$ th power divisor of $x$ and $y$. It is immediate from lemma 2.1 of [3] that, if $g(n)$ and $h(n)$ are multiplicative, then $S^{(k)}(n, r)$ is multiplicative in both variables $n$ and $r$.

For a given pair $n, r$ of positive integers we write $\hat{n}$ (resp $\hat{r}$ ) to denote the largest divisor of $n$ (resp $r$ ) that is relatively prime to $r$ (resp $n$ ). We write $\bar{n}$ for $\frac{\mathrm{n}}{\hat{\mathrm{n}}}$ and $\overline{\mathrm{r}}$ for $\frac{\mathrm{r}}{\hat{\mathrm{r}}}$. We prove the following

THEOREM. If $g(n)$ is completely multiplicative and $h(n)$ is multiplicative then

$$
\sum_{d^{k} \mid n}\left|S_{g, h}^{(k)}\left(d^{k}, r\right)\right|=\tau_{k}(\hat{n})\left|g\left(r_{*}\right)\right| \prod_{p \mid r}\left(|h(p)|+c_{k}|g(p)-h(p)|\right)
$$

or 0 according as $r_{*}^{k} \mid n$ or not, where $\tau_{k}(n)$ is the number of positive $k$ th power divisors of $n$ and the non negative integer $c_{k}=c_{k}(p)$ is determined so that

$$
\mathrm{p}^{\mathrm{kc}} \mathrm{k} \mid \frac{\mathrm{n}}{\mathrm{r}_{*}^{\mathrm{k}}} \text { and } \mathrm{p}^{\mathrm{k}\left(\mathrm{c}_{\mathrm{k}}+1\right)} \frac{\mathrm{n}}{\mathrm{r}_{\star}^{\mathrm{k}}} \text {. }
$$

We need the following lemmas.

LEMMA 1. For a prime $\mathrm{p}$ and non negative integers $\mathrm{a}$ and $\mathrm{b}$ we have

$$
\mathrm{s}^{(k)}\left(\mathrm{p}^{\mathrm{a}}, \mathrm{p}^{\mathrm{b}}\right)=\left\{\begin{array}{l}
1 \quad \text { if } \mathrm{b}=0 \\
\mathrm{~g}\left(\mathrm{p}^{\mathrm{b}}\right)-\mathrm{g}\left(\mathrm{p}^{\mathrm{b}-1}\right) \mathrm{h}(\mathrm{p}) \quad \text { if } \quad \mathrm{a} \geq \mathrm{bk} \geq \mathrm{k} \\
-\mathrm{g}\left(\mathrm{p}^{\mathrm{b}-1}\right) \mathrm{h}(\mathrm{p}) \quad \text { if } 0 \leq(\mathrm{b}-1) \mathrm{k} \leq \mathrm{a}<\mathrm{bk} \\
0 \quad \text { if } 0 \leq \mathrm{a}<(\mathrm{b}-1) \mathrm{k}
\end{array}\right.
$$

PROOF. Let $\left(p^{a}, p^{b k}\right)_{k}=p^{u k}$ so that $0 \leq u \leq b$. From (2.1) we have

$$
S^{(k)}\left(p^{a}, p^{b}\right)=\sum_{j=0}^{u} g\left(p^{j}\right) \mu\left(p^{b-j}\right) h\left(p^{b-j}\right) .
$$

If $b=0$ then $u=0$ and the r.h.s. of (2.3) is 1 while if $b \geq 1$ one has $\mathrm{u}=\mathrm{b}, \mathrm{u}=\mathrm{b}-1$ or $\mathrm{u} \leq \mathrm{b}-2$ according as $\mathrm{a} \geq \mathrm{bk},(\mathrm{b}-1) \mathrm{k} \leq \mathrm{a}<\mathrm{bk}$ or $\mathrm{a}<(\mathrm{b}-1) \mathrm{k}$ so that the r.h.s. of (2.3) has the value as stated in (2.2).

REMARK. If $g$ is completely multiplicative and $a \geq b k \geq k$ we have $S^{(k)}\left(p^{a}, p^{b}\right)=(g(p))^{b-1}(g(p)-h(p))$.

LEMMA 2. For each integer $i, 1 \leq i \leq s$, let $t_{i}$ be a non negative integer and for each ordered pair $(i, j), 1 \leq i \leq s, 0 \leq j \leq t_{i}$, let $a_{i j}$ be a complex number. Then

$$
\sum a_{1 j_{1}} a_{2 j_{2}} \ldots a_{s j_{s}}=\prod_{i=1}^{\pi}\left(a_{i 0}+a_{i 1}+\ldots+a_{i t_{i}}\right)
$$

where the summation on the left is extended over all s-tuples $\left(j_{1}, j_{2}, \ldots j_{s}\right)$ with $0 \leq j_{i} \leq t_{i}$. 
exactly once in the expansion of the product on the right and vice versa.

PROOF OF THE THEOREM. Let $n=\prod_{p \mid n} p^{a}\left(a=a_{p}\right)$ and $r=\prod_{p \mid r} p^{b}\left(b=b_{p}\right)$

be the canonical decompositions of $n$ and $r$ respectively.

Case (i) Suppose $r_{\star}^{k}+n$. In this case we have either $a<k(b-1)$ for some prime $p \mid \bar{r}$ or $b>1$ for some prime $p \mid \hat{r}$. Let $d^{k} \mid n$ and $d=\prod_{p \mid n} p^{\alpha}$ with $0 \leq \alpha \leq\left[\frac{a}{k}\right]$. Then clearly $r_{*}^{k}+d^{k}$ and hence we have either $0 \leq \alpha k<(b-1) k$ for some $p / \bar{r}$ or $b>1$ for some $p \mid \hat{r}$. This implies that $s^{(k)}\left(d^{k}, r\right)=0$ for each $k$ th power divisor $d^{k}$ of $n$ in virtue of lemma 1 and the multiplicativity of $s^{(k)}(n, r)$ in both variables $n$ and $r$.

Case (ii) Suppose $r_{*}^{k} \mid n$. In this case $a \geq k(b-1)$ for each $p \mid \bar{r}$ and $b=1$ for each $\mathrm{p} \mid \hat{\mathrm{r}}$. Let $\mathrm{d}^{\mathrm{k}} \mid \mathrm{n}$. Then $\mathrm{d}$ can be uniquely expressed as $\overline{\mathrm{d}} \hat{\mathrm{d}}$ with $\bar{d}^{\mathrm{k}}\left|\overline{\mathrm{n}}, \hat{d}^{\mathrm{k}}\right| \hat{\mathrm{n}}$ and $(\bar{d}, \hat{d})=1$. The multiplicativity of $s^{(k)}(n, r)$ in both variables implies

$$
\begin{aligned}
S^{(k)}\left(d^{k}, r\right) & =s^{(k)}\left(\bar{d}^{k} \hat{d}^{k}, \bar{r} \hat{r}\right) \\
& =S^{(k)}\left(\bar{d}^{k}, \bar{r}\right) S^{(k)}\left(\hat{d}^{k}, 1\right) S^{(k)}(1, \hat{r}) \\
& =\mu(\hat{r}) h(\hat{r}) S^{(k)}\left(\bar{d}^{k}, \bar{r}\right) .
\end{aligned}
$$

Hence we have

$$
\sum_{\mathrm{d}^{k} \mid \mathrm{n}}\left|\mathrm{S}^{(\mathrm{k})}\left(\mathrm{d}^{\mathrm{k}}, \mathrm{r}\right)\right|=\tau_{\mathrm{k}}(\hat{\mathrm{n}})|\mathrm{h}(\hat{\mathrm{r}})| \sum_{\mathrm{d}^{k} \mid \overline{\mathrm{n}}}\left|\mathrm{S}^{(\mathrm{k})}\left(\mathrm{d}^{\mathrm{k}}, \overline{\mathrm{r}}\right)\right|
$$

since, for a given $k$ th power divisor $x^{k}$ of $\bar{n}$, the number of $k$ th power divisors $d^{k}$ of $n$ for which $\bar{d}=x$ is $\tau_{k}(\hat{n})$. Again in virtue of the multiplicativity of $s(k)(n, r)$ in both variables we have

$$
\sum_{d^{k} \mid \bar{n}}\left|s^{(k)}\left(d^{k}, \bar{r}\right)\right|=\sum\left(\prod_{i=1}^{s}\left|s^{(k)}\left(p_{i}^{k p_{i}}, p_{i}{ }^{b}\right)\right|\right)
$$

where $p_{1}, p_{2} \cdots p_{s}$ are the prime divisors of $\bar{n}$ (hence of $\bar{r}$ ), $p_{i}{ }^{b}\left\|\bar{r}, p_{i}{ }^{a}\right\| \bar{n}$ and the summation on the right is extended over all s-tuples $\left(\ell_{1}, \ell_{2}, \ldots \ell_{s}\right)$ with $0 \leq \ell_{i} \leq\left[\frac{a_{i}}{k}\right]$. Now lemma 2 implies that

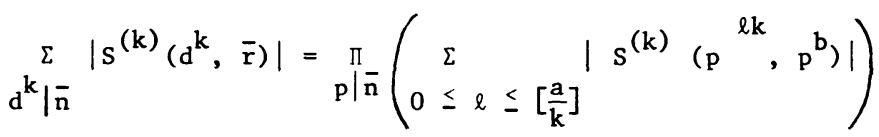

$$
\begin{aligned}
& =\prod_{\mathrm{p} \mid \overline{\mathrm{r}}}\left|\mathrm{g}\left(\mathrm{p}^{\mathrm{b}-1}\right)\right|\left\{|\mathrm{h}(\mathrm{p})|+\left(\left[\frac{\mathrm{a}}{\mathrm{k}}\right]-\mathrm{b}+1\right)|\mathrm{g}(\mathrm{p})-\mathrm{h}(\mathrm{p})|\right\} \\
& =\left|g\left(r_{*}\right)\right| \prod_{p \mid \bar{r}}\left(|h(p)|+c_{k}|g(p)-h(p)|\right)
\end{aligned}
$$

The conclusion in this case now follows in virtue of $(2.5)$, on pushing $|h(\hat{r})|$ into the product (since $\mathrm{p} \mid \hat{\mathrm{r}} \Longrightarrow \mathrm{c}_{\mathrm{k}}(\mathrm{p})=0$ ).

3. FORMULAE FOR RAMANUJAN'S SUM AND ITS GENERALIZATIONS.

Let $f=f(x)$ be a polynomial of positive degree with integer coefficients and, for positive integral $r$, let $\mathrm{N}_{\mathrm{f}}(\mathrm{r}$ ) denote the number of incongruent solutions (mod $r$ ) of the congruence $f(x) \equiv 0(\bmod r)$. Suppose such a polynomial $f$, a multiplicative arithmetical function $n(n)$ and positive integers $k$ and $t$ are given. Then, by taking $g(n)=n k t$ and $h(n)=n(n) N_{f}^{t}\left(n^{k}\right)\left(N_{f}^{t}(r)=\left(N_{f}(r)\right)^{t}\right)$ in (2.1) we have the generalized Ramanujan's 
sum introduced by Chidambaraswamy [3], viz.,

$$
\left.C_{f, t}^{k, n}(n, r)=d^{k} \sum_{(n, r}^{k}\right)_{k}^{k t} \mu\left(\frac{r}{d}\right) \quad n\left(\frac{r}{d}\right) N_{f}^{t}\left(\frac{r^{k}}{d^{k}}\right) .
$$

This function includes as special cases (see [3]) the Ramanujan's sum $c(n, r)$ and some of its generalizations. In fact, writing $I(n)=1$ for all $n$ and, for a given positive integer $u, \mu_{u}(n)=\exp \left(\Pi i \omega(n) u^{-1}\right)$ or 0 according as $n$ is or is not squarefree $(\omega(n)$ being the number of distinct prime factors of $n)$, we have $c_{x, 1}^{k, I}(n, r)=C^{(k)}(n, r)$ (Cohen [4]), $\quad C_{x, t}^{1, I}(n, r)=C_{t}(n, r) \quad($ Cohen $[5]), C_{x, t}^{k, I}(n, r)=C_{t}^{(k)}(n, r)$ (M. Sugunamma [6]) and $\mathrm{C}_{\mathrm{x}, 1}^{1, \mu \mu} \mathrm{u}(\mathrm{n}, \mathrm{r})=\mathrm{C}^{\mu} \mathrm{u}(\mathrm{n}, \mathrm{r})$ (C. S. Venkataraman and R. Sivaramakrishnan [7]).

Specializing the functions $g(n)$ and $h(n)$ suitably in our theorem we obtain, for the functions described above, the following formulae:

$$
\begin{aligned}
& \sum_{d^{k} \mid n}\left|C_{f, t}^{k, n}\left(d^{k}, r\right)\right|=r_{*}^{k t} \tau_{k}(\hat{n}) \prod_{p \mid r}\left(\left|n(p) N_{f}^{t}\left(p^{k}\right)\right|+c_{k}\left|p^{k t}-n(p) N_{f}^{t}\left(p^{k}\right)\right|\right) \\
& \text { or } 0 \text { according as } \mathrm{r}_{*}^{\mathrm{k}} \mid \mathrm{n} \text { or not } \\
& \sum_{\mathrm{d} \mid \mathrm{n}}\left|\mathrm{c}^{\mu} \mathrm{u}(\mathrm{d}, \mathrm{r})\right|=\mathrm{r}_{\star} \tau(\hat{\mathrm{n}}) \prod_{\mathrm{p} \mid \mathrm{r}}\left(1+\mathrm{c}_{1}\left|\mathrm{p}-\exp \left(\pi i \mathrm{u}^{-1}\right)\right|\right) \\
& \text { or } 0 \text { according as } r_{\star} \mid n \text { or not } \\
& \sum_{\mathrm{d}^{k} \mid \mathrm{n}}\left|\mathrm{c}_{\mathrm{t}}^{(\mathrm{k})}\left(\mathrm{d}^{\mathrm{k}}, \mathrm{r}\right)\right|=\mathrm{r}_{\star}^{\mathrm{kt}} \tau_{\mathrm{k}}(\hat{\mathrm{n}}) \prod_{\mathrm{p} \mid \mathrm{r}}\left(1+\mathrm{c}_{\mathrm{k}}\left(\mathrm{p}^{\mathrm{kt}}-1\right)\right) \text { or } 0 \\
& \text { according as } r_{*}^{k} \mid n \text { or not } \\
& \mathrm{d}^{\mathrm{k}} \sum_{\mathrm{n}}\left|\mathrm{C}(\mathrm{k})\left(\mathrm{d}^{\mathrm{k}}, \mathrm{r}\right)\right|=\mathrm{r}_{*}^{\mathrm{k}} \tau_{\mathrm{k}}(\hat{\mathrm{n}}) \prod_{\mathrm{p} \mid \mathrm{r}}\left(1+\mathrm{c}_{\mathrm{k}}\left(\mathrm{p}^{\mathrm{k}}-1\right)\right) \text { or } 0 \text { according } \\
& \text { as } r_{*}^{k} / n \text { or not }
\end{aligned}
$$

Clearly (3.7) is the same as (1.1).

\section{REFERENCES}

1. Johnson, K. R. A Result For the 'Other' Variable of the Ramanujan's Sum, Elem. Math. 38 (1983), 122-123.

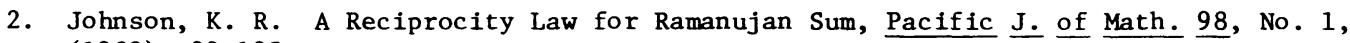
(1982), 99-105.

3. Chidambaraswamy, J. Generalized Ramanujan's Sum, Period. Math. Hungar., 10(1), (1979), 71-87.

4. Cohen, E. An Extension of Ramanujan's Sum, Duke Math. J. 16 (1949), 85-90

5. Cohen, E. Trigonometric Sums in Elementary Number Theory, Amer. Math. Monthly 66 (1959) , 105-117.

6. Sugunamma, M. Eckford Cohen's Generalization of Ramanujan's Trigonometrical Sum $C(n, r)$, Duke Math. J. 27 (1960), 323-330.

7. Venkataraman, C. S. and Sivaramakrishnan, R. An Extension of Ramanujan's Sum, Math. Student 40A (1972), 211-216. 


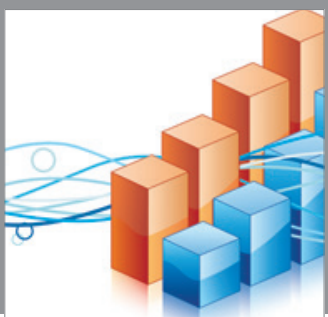

Advances in

Operations Research

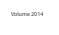

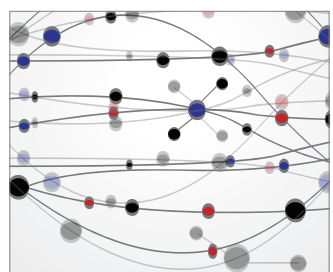

\section{The Scientific} World Journal
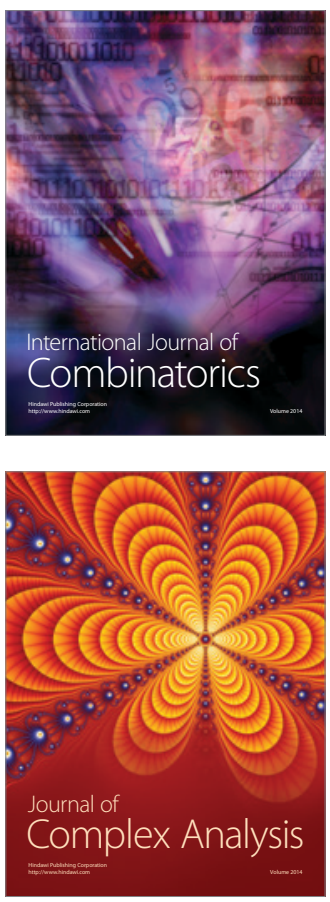

International Journal of

Mathematics and

Mathematical

Sciences
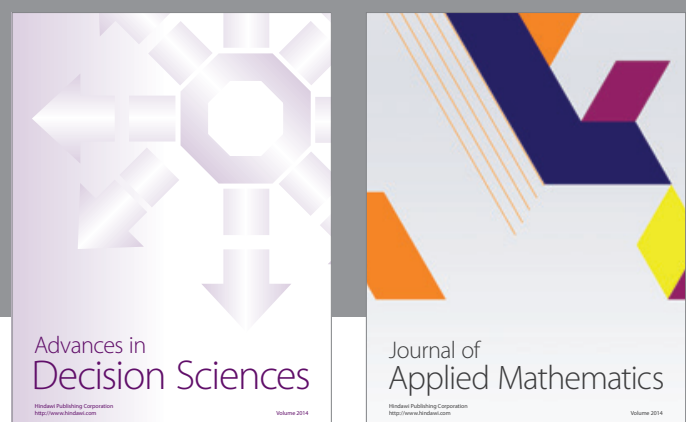

Journal of

Applied Mathematics
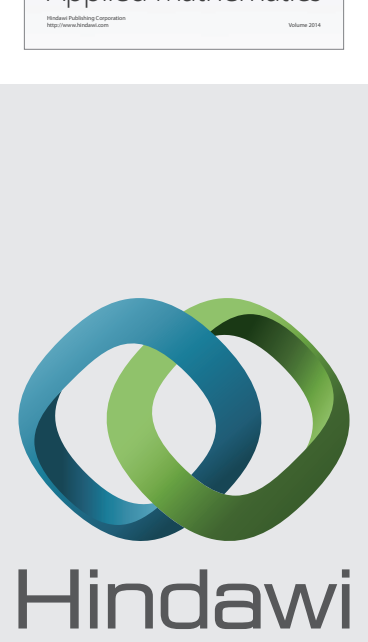

Submit your manuscripts at http://www.hindawi.com
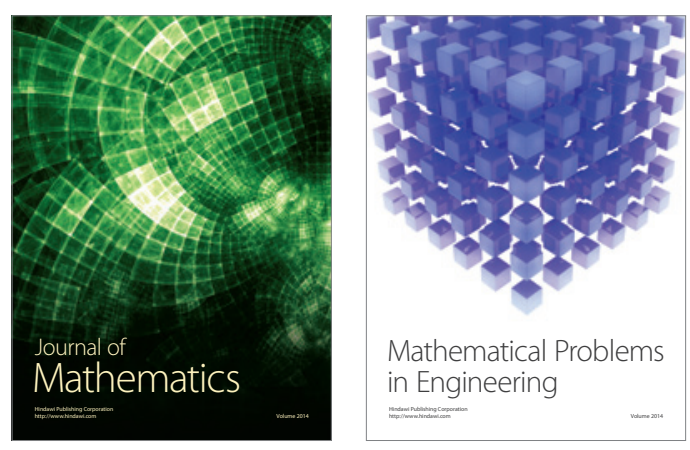

Mathematical Problems in Engineering
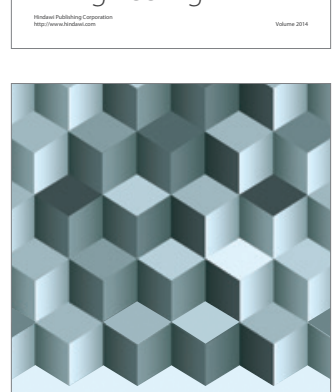

Journal of

Function Spaces
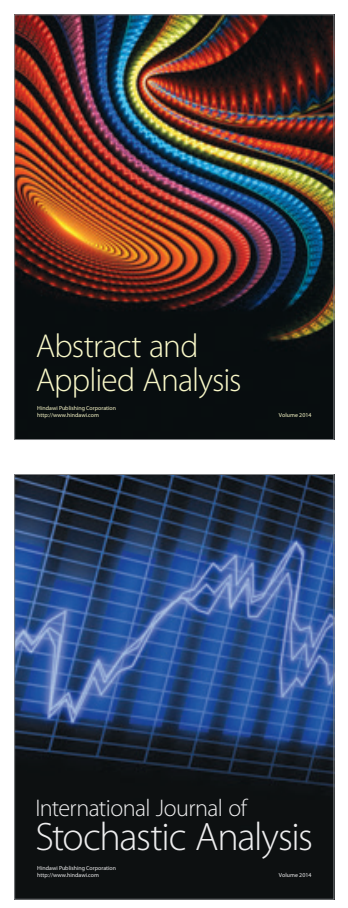

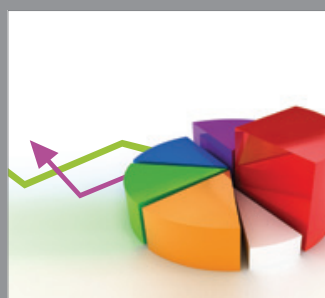

ournal of

Probability and Statistics

Promensencen
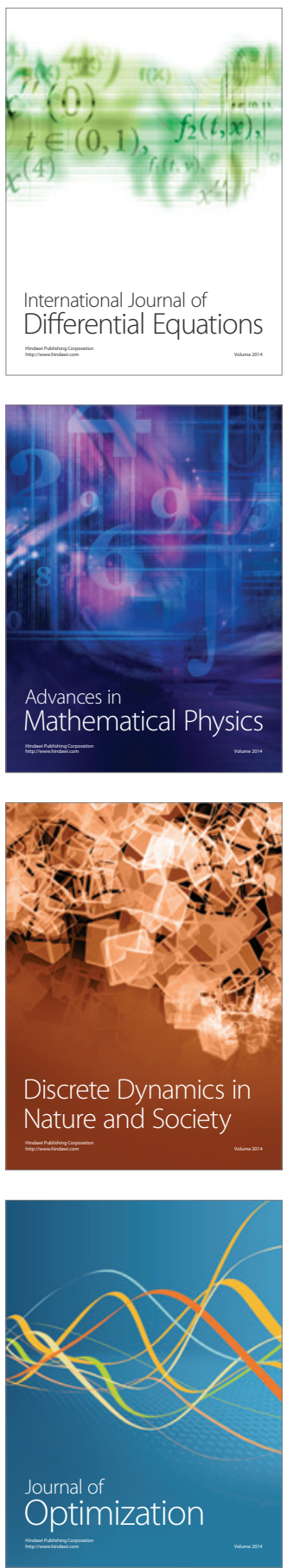